MS\#JO061533E-Supporting Information-Revised

\title{
Thermo-solvatochromism of Merocyanine Polarity Indicators in Pure and Aqueous Solvents: Relevance of Solvent Lipophilicity
}

\author{
Clarissa T. Martins, Michelle S. Lima, and Omar A. El Seoud, \\ Instituto de Química, Universidade de São Paulo, \\ C.P. 26077, 05513-970, São Paulo, S.P., Brazil, \\ elseoud@iq.usp.br
}

\section{Table of Contents}

Figure SI-1. Plots of $\mathrm{E}_{\mathrm{T}}\left(\mathrm{OcPMBr}_{2}\right)$ versus the water mole fraction $\chi_{\mathrm{w}}$, for different binary mixtures

Figure SI-2. Solvent polarity/temperature/solvent composition contours for $\mathrm{MePMBr}_{2}$, $\mathrm{BuPMBr}_{2}$ and $\mathrm{OcPMBr}_{2}$ in (A), $\mathrm{PrOH} / \mathrm{W}$, (B) $\mathrm{MeCN} / \mathrm{W}$, (C) DMSO / W

Table SI-1. Results of the application of Eqn (6): $E_{T}($ probe $)=$ Constant $+s\left(\pi^{*}\right.$ solv $\left.+d \delta\right)+a$ $\alpha_{\text {solv }}+b \beta_{\text {solv }}+p \log P_{\text {solv }}$

Table SI-2. Thermo-solvatochromic data for $\mathrm{MePMBr}_{2}, \mathrm{BuPMBr}_{2}$ and $\mathrm{OctPMBr}_{2}$ in binary solvent mixtures. Polynomial dependence of $\mathrm{E}_{\mathrm{T}}\left(\mathrm{RPMBr}_{2}\right), \mathrm{kcal} / \mathrm{mol}$, on the analytical mole fraction of water in the binary mixture, $x_{\mathrm{w}}^{\text {Analytical }}$

Table SI-3. Regression cofficients of Eqn. 14, applied to solvatochromism of the probes synthesized in aqueous methanol and 1-propanol, respectively, at $25^{\circ} \mathrm{C}$

Table SI-4. Yields, elemental analyses and relevant IR frequencies of the probes synthesized

Table SI-5. Structures, numbering of protons and ${ }^{1} \mathrm{H}$ NMR data for the probes synthesized

Table SI-6. Structures, numbering carbons and ${ }^{13} \mathrm{C}$ NMR data for the probes synthesized 
Table of Contents, continue

Calculations SI-1. Dependence of $E_{T}$ (probe) on the properties of the $S 13$ solvent.....................

Calculations SI-2. Calculation of the dissociation constant of Solv-W, $\mathrm{K}_{\text {dissoc }}, x_{\text {Species }}^{\text {Effective }}$, and

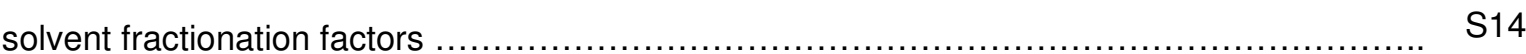

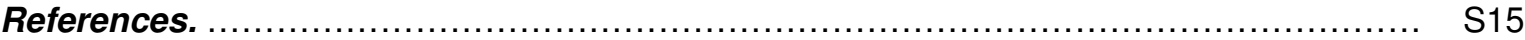



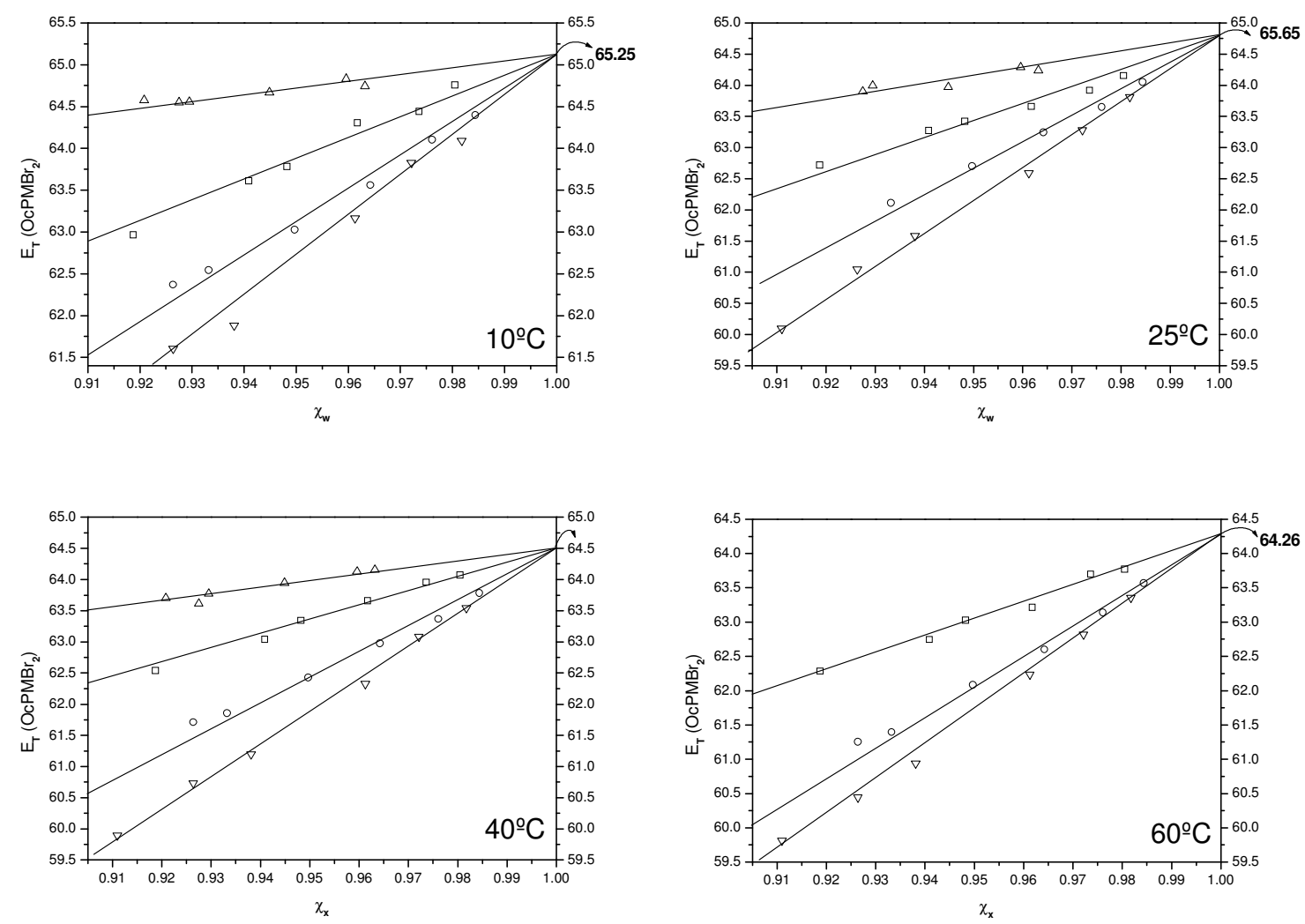

Figure SI-1. Plots of $\mathrm{E}_{\mathrm{T}}\left(\mathrm{OCPMBr}_{2}\right)$ versus the water mole fraction $\chi_{\mathrm{w}}$, for different binary mixtures. Each sub-Figure corresponds to a fixed temperature. The mixtures were: $(\Delta)$, $\mathrm{MeOH}-\mathrm{W}$; $(\square)$, MeCN-W; (O), Acetone-W; $(\nabla), \mathrm{PrOH}-\mathrm{W}$ 
B - PrOH / W
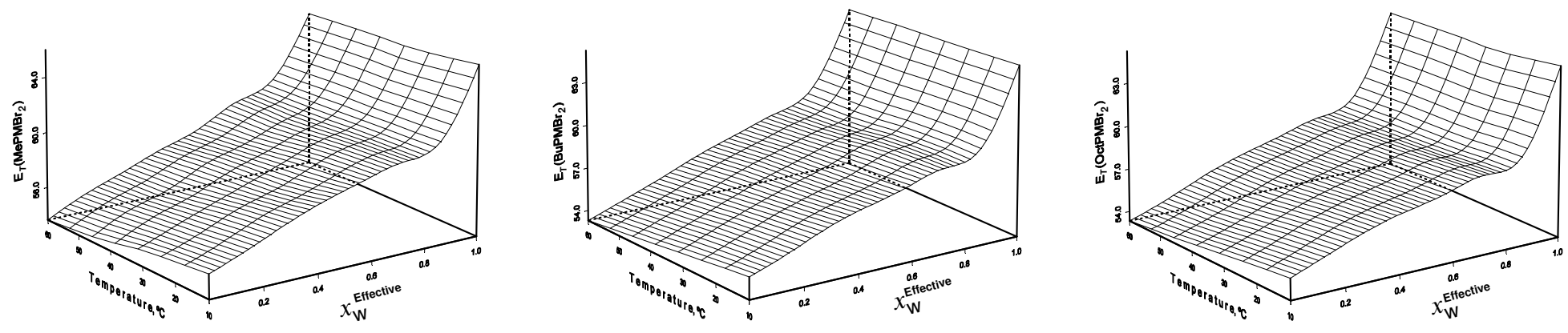

$\mathrm{C}-\mathrm{MeCN} / \mathrm{W}$
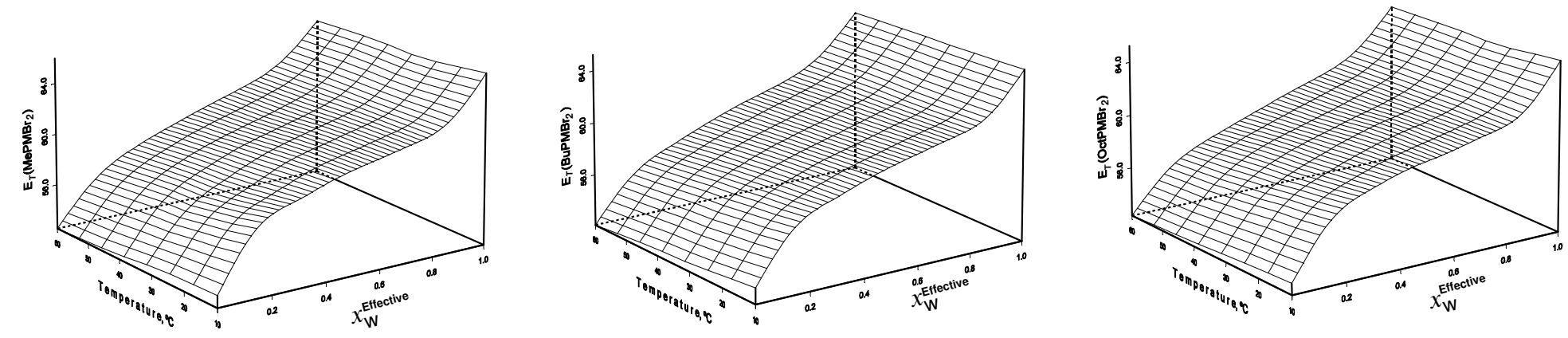

Figure SI-2 - Solvent polarity/temperature/solvent composition contours for $\mathrm{MePMBr}_{2}, \mathrm{BuPMBr}_{2}$ and $\mathrm{OcPMBr}_{2}$ in $(\mathrm{A})$, $\mathrm{PrOH} / \mathrm{W}$, (B) $\mathrm{MeCN} / \mathrm{W}$, (c) DMSO / W. 
Figure SI-2, continue.

D - DMSO / W
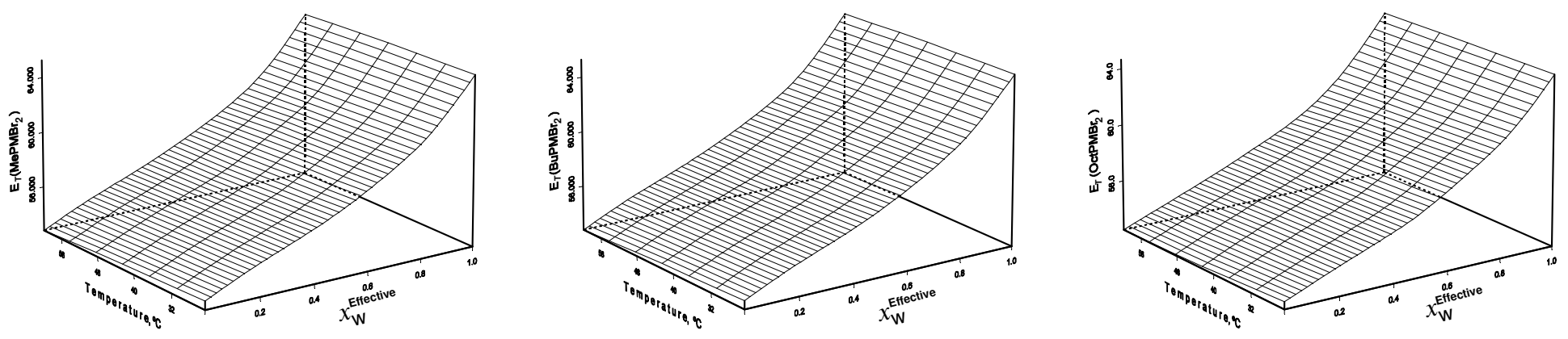
Table SI-1. Results of the application of Eqn (6): $\mathrm{E}_{\mathrm{T}}($ probe $)=$ Constant $+\mathrm{s}\left(\pi_{\text {solv }}^{*}+\mathrm{d} \delta\right)+$ $a \alpha_{\text {solv }}+b \beta_{\text {solv }}+p \log P_{\text {solv }}$

\begin{tabular}{|c|c|c|c|c|c|}
\hline probe & constant & $S$ & $a$ & $b$ & $p$ \\
\hline $\mathrm{MePMBr}_{2}$ & 42.73 & 9.61 & 8.79 & 0.35 & -0.89 \\
\hline $\mathrm{BuPMBr}_{2}$ & 42.98 & 9.32 & 8.57 & -0.14 & -0.76 \\
\hline $\mathrm{OcPMBr}_{2}$ & 43.04 & 9.23 & 8.47 & 0.08 & -0.78 \\
\hline $\mathrm{RB}$ & 31.72 & 13.24 & 14.43 & -0.24 & -0.34 \\
\hline WB & 40.97 & 13.13 & 14.05 & -0.73 & -0.46 \\
\hline QB & 48.36 & 6.21 & 8.58 & 0.17 & -0.08 \\
\hline MePM & 41.28 & 8.87 & 11.30 & -1.09 & -0.56 \\
\hline $\mathrm{BuQMBr}_{2}$ & 41.61 & -2.89 & 4.49 & -0.90 & -0.99 \\
\hline P1 & 37.16 & 8.62 & 12.35 & -2.24 & -1.12 \\
\hline P2 & 26.38 & 14.36 & 12.62 & 1.83 & 0.48 \\
\hline P3 & 51.72 & -3.75 & -1.27 & -0.42 & 0.02 \\
\hline P4 & 76.36 & -7.43 & -1.86 & 2.05 & 0.25 \\
\hline P5 & 78.73 & -7.43 & -1.93 & 1.28 & 0.26 \\
\hline P6 & 85.22 & 3.48 & 5.74 & -0.83 & 0.19 \\
\hline P7 & 100.46 & -6.01 & -0.51 & -7.14 & -0.11 \\
\hline P8 & 82.40 & -7.48 & -1.12 & -4.52 & 0.06 \\
\hline P9 & 82.30 & -8.09 & -0.77 & -4.14 & 0.42 \\
\hline P10 & 80.86 & -6.91 & -1.14 & -3.70 & 0.25 \\
\hline P11 & 77.05 & -7.70 & -1.26 & 0.59 & 0.26 \\
\hline P12 & 87.33 & -6.12 & -0.88 & -8.92 & 0.09 \\
\hline P13 & 54.45 & 14.94 & 19.53 & -0.74 & 0.11 \\
\hline
\end{tabular}


Table SI-2. Thermo-solvatochromic data for $\mathrm{MePMBr}_{2}, \mathrm{BuPMBr}_{2}$ and $\mathrm{OctPMBr}_{2}$ in binary solvent mixtures. The polynomial dependence of the observed $\mathrm{E}_{\mathrm{T}}\left(\mathrm{RPMBr}_{2}\right), \mathrm{kcal} / \mathrm{mol}$, on the analytical mole fraction of water in the binary mixture, $x_{\mathrm{w}}^{\text {Analytical }}$, has been calculated according to the equation: $E_{T}\left(R P M B r_{2}\right)=E_{T}\left(R P M B r_{2}\right)_{S o l v}+B\left(\chi_{W}\right)+C\left(\chi_{W}\right)^{2}+D\left(\chi_{W}\right)^{3}+E\left(\chi_{W}\right)^{4}+F\left(\chi_{W}\right)^{5}+G(\chi$ w) $)^{6}$.

\begin{tabular}{|c|c|c|c|c|c|c|c|c|c|c|}
\hline $\begin{array}{c}\text { Binary } \\
\text { mixture/Probe }\end{array}$ & $\begin{array}{l}\mathrm{T}, \\
{ }^{\circ} \mathrm{C}^{\mathrm{d}}\end{array}$ & $\mathrm{E}_{\mathrm{T}}\left(\mathrm{RPMBr}_{2}\right)_{\text {Solv }^{\prime}}{ }^{\mathrm{e}}$ & B & C & $\mathrm{D}$ & $\mathrm{E}$ & $\mathrm{F}$ & $\mathrm{G}$ & $r^{2 t}$ & $S^{f}$ \\
\hline \multicolumn{11}{|l|}{$\mathrm{MeOH} / \mathrm{W}$} \\
\hline \multirow[t]{3}{*}{$\mathrm{MePMBr}_{2}$} & 10 & 59.775 [0.038] & 3.118 & 0.638 & -1.155 & 3.557 & & & 0.9996 & 0.045 \\
\hline & 25 & 59.277 [0.025] & 2.689 & 2.741 & -2.973 & 3.666 & & & 0.9998 & 0.030 \\
\hline & 40 & 58.592 [0.051] & 4.197 & 2.081 & -6.056 & 6.264 & & & 0.9994 & 0.061 \\
\hline \multirow[t]{3}{*}{$\mathrm{BuPMBr}_{2}$} & 10 & $59.235[0.076]$ & 4.069 & -5.563 & 9.995 & -2.389 & & & 0.9986 & 0.090 \\
\hline & 25 & 58.650 [0.050] & 3.876 & -2.088 & 3.116 & 1.479 & & & 0.9994 & 0.060 \\
\hline & 40 & $58.079[0.049]$ & 3.691 & 0.146 & -0.194 & 3.036 & & & 0.9995 & 0.058 \\
\hline \multirow[t]{3}{*}{$\mathrm{OcPMBr}_{2}$} & 10 & $59.028[0.062]$ & 3.912 & -1.251 & 1.825 & 1.817 & & & 0.9991 & 0.073 \\
\hline & 25 & $58.491[0.066]$ & 3.974 & 1.741 & 2.544 & 1.471 & & & 0.9989 & 0.078 \\
\hline & 40 & 52.927 [0.055] & 3.952 & -0.127 & 0.017 & 2.815 & & & 0.9993 & 0.064 \\
\hline \multicolumn{11}{|l|}{$\mathrm{PrOH} / \mathrm{W}$} \\
\hline \multirow[t]{4}{*}{$\mathrm{MePMBr}_{2}$} & 10 & $55.424[0.100]$ & -0.955 & 94.363 & -406.165 & 833.352 & -828.008 & 318.062 & 0.9992 & 0.107 \\
\hline & 25 & $54.974[0.081]$ & -0.214 & 92.862 & -444.562 & 971.403 & -984.356 & 375.358 & 0.9995 & 0.087 \\
\hline & 40 & $54.256[0.109]$ & 4.403 & 48.128 & -258.003 & 609.220 & -656.479 & 263.676 & 0.9991 & 0.116 \\
\hline & 60 & $53.647[0.082]$ & 4.485 & 63.212 & -357.625 & 839.058 & -876.125 & 338.204 & 0.9995 & 0.087 \\
\hline \multirow[t]{2}{*}{$\mathrm{BuPMBr}_{2}$} & 10 & $54.886[0.169]$ & 0.616 & 74.710 & -349.231 & 757.630 & -779.861 & 306.679 & 0.9978 & 0.176 \\
\hline & 25 & $54.433[0.131]$ & 1.306 & 79.442 & -404.617 & 907.590 & -934.571 & 361.498 & 0.9987 & 0.136 \\
\hline
\end{tabular}


Table SI-2, continue

\begin{tabular}{|c|c|c|c|c|c|c|c|c|c|c|}
\hline & 40 & $53.948[0.093]$ & 1.500 & 72.217 & -357.231 & 799.461 & -831.772 & 326.628 & 0.9994 & 0.097 \\
\hline & 60 & $53.343[0.078]$ & 4.175 & 31.901 & -177.182 & 449.703 & -518.865 & 221.352 & 0.9996 & 0.081 \\
\hline \multirow[t]{4}{*}{$\mathrm{OcPMBr}_{2}$} & 10 & $54.916[0.170]$ & 1.913 & 70.208 & -373.745 & 867.097 & -925.282 & 370.311 & 0.9975 & 0.177 \\
\hline & 25 & $54.425[0.130]$ & 1.519 & 71.089 & -360.879 & 820.207 & -867.579 & 345.985 & 0.9986 & 0.135 \\
\hline & 40 & $53.948[0.110]$ & 0.752 & 72.837 & -358.738 & 812.083 & -858.184 & 341.822 & 0.9990 & 0.115 \\
\hline & 60 & $53.382[0.107]$ & -0.300 & 89.520 & -422.473 & 920.262 & -944.192 & 368.053 & 0.9992 & 0.111 \\
\hline \multicolumn{11}{|l|}{$M e C N / W$} \\
\hline \multirow[t]{4}{*}{$\mathrm{MePMBr}_{2}$} & 10 & $53.567[0.078]$ & 42.838 & -144.935 & 293.846 & -300.546 & 121.164 & & 0.9995 & 0.087 \\
\hline & 25 & $53.410[0.104]$ & 31.799 & -82.761 & 154.633 & -159.386 & 67.702 & & 0.9992 & 0.116 \\
\hline & 40 & $52.950[0.050]$ & 31.789 & -81.537 & 148.304 & -148.094 & 61.777 & & 0.9998 & 0.056 \\
\hline & 60 & $52.550[0.043]$ & 29.334 & -72.459 & 131.777 & -130.332 & 53.946 & & 0.9999 & 0.049 \\
\hline \multirow[t]{4}{*}{$\mathrm{BuPMBr}_{2}$} & 10 & $53.539[0.064]$ & 36.897 & -122.025 & 258.555 & -277.054 & 115.566 & & 0.9997 & 0.071 \\
\hline & 25 & $52.907[0.044]$ & 34.315 & -102.431 & 207.808 & -220.851 & 93.238 & & 0.9999 & 0.050 \\
\hline & 40 & $52.569[0.037]$ & 31.320 & -83.544 & 161.200 & -169.262 & 72.432 & & 0.9999 & 0.042 \\
\hline & 60 & $52.081[0.043]$ & 30.610 & -87.324 & 180.956 & -194.335 & 82.449 & & 0.9999 & 0.048 \\
\hline \multirow[t]{4}{*}{$\mathrm{OcPMBr}_{2}$} & 10 & $53.255[0.074]$ & 38.829 & -127.402 & 262.053 & -278.312 & 116.764 & & 0.9995 & 0.083 \\
\hline & 25 & $53.002[0.063]$ & 33.121 & -98.504 & 202.707 & -219.815 & 94.235 & & 0.9997 & 0.071 \\
\hline & 40 & $52.688[0.060]$ & 28.075 & -66.826 & 122.643 & -130.071 & 57.989 & & 0.9998 & 0.065 \\
\hline & 60 & $52.275[0.070]$ & 27.796 & -77.092 & 160.486 & -174.023 & 74.804 & & 0.9997 & 0.078 \\
\hline \multicolumn{11}{|l|}{$D M S O$ / W } \\
\hline \multirow[t]{3}{*}{$\mathrm{MePMBr}_{2}$} & 25 & $53.421[0.071]$ & 4.741 & 5.667 & 1.280 & & & & 0.9996 & 0.094 \\
\hline & 40 & $53.207[0.079]$ & 4.743 & 5.061 & 1.953 & & & & 0.9995 & 0.103 \\
\hline & 60 & $52.833[0.086]$ & 5.347 & 3.785 & 2.648 & & & & 0.9994 & 0.113 \\
\hline
\end{tabular}


Table SI-2, continue

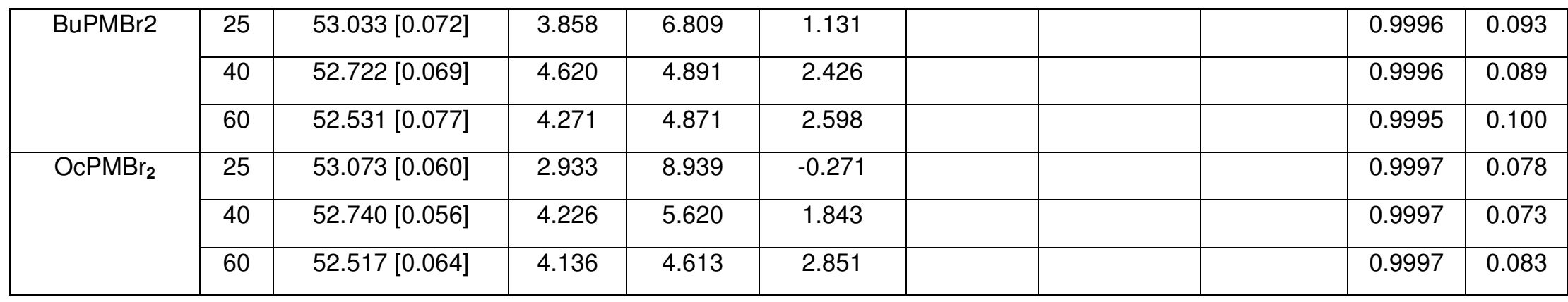

d- The temperature range investigated was dictated by the b.p. (MeOH) or the m.p. (DMSO) of the solvent.

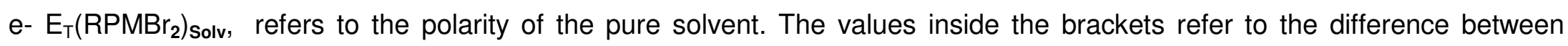
calculated and experimental $\mathrm{E}_{\mathrm{T}}\left(\mathrm{RPMBr}_{2}\right)^{\text {Solvent }}$

$\mathrm{f}$ - Abbreviations: $\mathrm{r}^{2}=$ correlation coefficient for the polynomial regression, $\mathrm{SD}=$ standard deviation.

Table SI-3. Regression cofficients of Eqn. 14, applied to solvatchromism of the probes synthesized in aqueous methanol and 1propanol, respectively, at $25^{\circ} \mathrm{C}$.

\begin{tabular}{|c|c|c|c|c|c|c|}
\hline mixture & probe & constant & s & a & $b$ & $p$ \\
\hline \multirow{3}{*}{$\mathbf{M e O H} / \boldsymbol{W}$} & $\mathrm{MePMBr}_{2}$ & 66.6 & -9.70 & -0.15 & -12.45 & -12.99 \\
\cline { 2 - 7 } & $\mathrm{BuPMBr}_{2}$ & 70.31 & -13.64 & -0.29 & -15.03 & -15.64 \\
\cline { 2 - 7 } & $\mathrm{OcPMBr}_{2}$ & 69.94 & -13.90 & -0.33 & -14.19 & -16.11 \\
\hline \multirow{2}{*}{$\mathbf{P r O H} \boldsymbol{W}$} & $\mathrm{MePMBr}_{2}$ & -109.48 & 160.38 & 5.04 & -14.93 & 50.60 \\
\cline { 2 - 7 } & $\mathrm{BuPMBr}_{2}$ & -117.16 & 164.38 & 5.58 & -19.75 & 52.42 \\
\cline { 2 - 7 } & $\mathrm{OcPMBr}_{2}$ & -141.41 & 193.33 & 6.122 & -22.358 & 63.32 \\
\hline
\end{tabular}


Table SI-4. Yields, elemental analyses and relevant IR frequencies of the probes synthesized.

\begin{tabular}{|c|c|c|c|c|c|c|}
\hline \multirow[t]{2}{*}{ Probe } & \multirow{2}{*}{$\begin{array}{l}\text { Molecular } \\
\text { Formula }\end{array}$} & \multirow{2}{*}{$\begin{array}{c}\text { Yield } \\
(\%)\end{array}$} & \multirow[t]{2}{*}{ m.p. $\left({ }^{\circ} \mathrm{C}\right)$} & \multicolumn{2}{|c|}{ Elemental Analyses } & \multirow[t]{2}{*}{$\mathrm{IR}\left(\mathrm{KBr}, \mathrm{cm}^{-1}\right)$} \\
\hline & & & & Calculated & Analyzed & \\
\hline $\mathrm{MePMBr}_{2}$ & $\mathrm{C}_{14} \mathrm{H}_{11} \mathrm{Br}_{2} \mathrm{NO}$ & 60 & $274-275$ & $\begin{array}{l}\% \text { C } 45.56 \\
\% H 3.19 \\
\% N 3.80\end{array}$ & $\begin{array}{l}\% \mathrm{C} 45.45 \\
\% \mathrm{H} 3.19 \\
\% \mathrm{~N} 3.63\end{array}$ & $\begin{array}{l}3032\left(v_{\mathrm{C}-\mathrm{H}}\right) ; \\
1604\left(v_{\mathrm{C}=\mathrm{C}}\right) ; \\
1173\left(v_{\mathrm{C}-\mathrm{N}}\right) ; \\
1038\left(v_{\mathrm{C}-\mathrm{Br}}\right) .\end{array}$ \\
\hline $\mathrm{BuPMBr}_{2}$ & $\mathrm{C}_{17} \mathrm{H}_{17} \mathrm{Br}_{2} \mathrm{NO}$ & 53 & $245-246$ & $\begin{array}{l}\% \mathrm{C} 49.66 \\
\% \mathrm{H} 4.17 \\
\% \mathrm{~N} 3.41\end{array}$ & $\begin{array}{l}\% \mathrm{C} 49.32 \\
\% \mathrm{H} 4.20 \\
\% \mathrm{~N} 3.21\end{array}$ & $\begin{array}{l}3041\left(v_{\mathrm{C}-\mathrm{H}}\right) \\
1563\left(v_{\mathrm{C}=\mathrm{C}}\right) \\
1170\left(v_{\mathrm{C}-\mathrm{N}}\right) \\
1039\left(v_{\mathrm{C}-\mathrm{Br}}\right)\end{array}$ \\
\hline $\mathrm{HxPMBr}_{2}$ & $\mathrm{C}_{19} \mathrm{H}_{21} \mathrm{Br}_{2} \mathrm{NO}$ & 65 & $242-243$ & $\begin{array}{l}\% \text { C } 51.96 \\
\% H 4.82 \\
\% N 3.19\end{array}$ & $\begin{array}{l}\text { \%C } 52.31 \\
\% H 5.32 \\
\% N ~ 3.14\end{array}$ & $\begin{array}{l}3036\left(v_{\mathrm{C}-\mathrm{H}}\right) ; \\
1558\left(\mathrm{v}_{\mathrm{C}=\mathrm{C}}\right) ; \\
1165\left(\mathrm{v}_{\mathrm{C}-\mathrm{N}}\right) ; \\
1040\left(\mathrm{v}_{\mathrm{C}-\mathrm{Br}}\right)\end{array}$ \\
\hline $\mathrm{OcPMBr}_{2}$ & $\mathrm{C}_{21} \mathrm{H}_{25} \mathrm{Br}_{2} \mathrm{NO}$ & 59 & $138-140$ & $\begin{array}{l}\% \text { C } 53.98 \\
\% H 5.39 \\
\% N 3.00\end{array}$ & $\begin{array}{l}\% \text { C } 53.52 \\
\% H 5.43 \\
\% N ~ 2.88\end{array}$ & $\begin{array}{l}3040\left(v_{\mathrm{C}-\mathrm{H}}\right) ; \\
1557\left(v_{\mathrm{C}=\mathrm{C}}\right) ; \\
1163\left(v_{\mathrm{C}-\mathrm{N}}\right) ; \\
1040\left(v_{\mathrm{C}-\mathrm{Br}}\right)\end{array}$ \\
\hline
\end{tabular}


Table SI-5. Structures, numbering of protons and ${ }^{1} \mathrm{H}$ NMR data for the probes synthesized. ${ }^{\text {a,b }}$

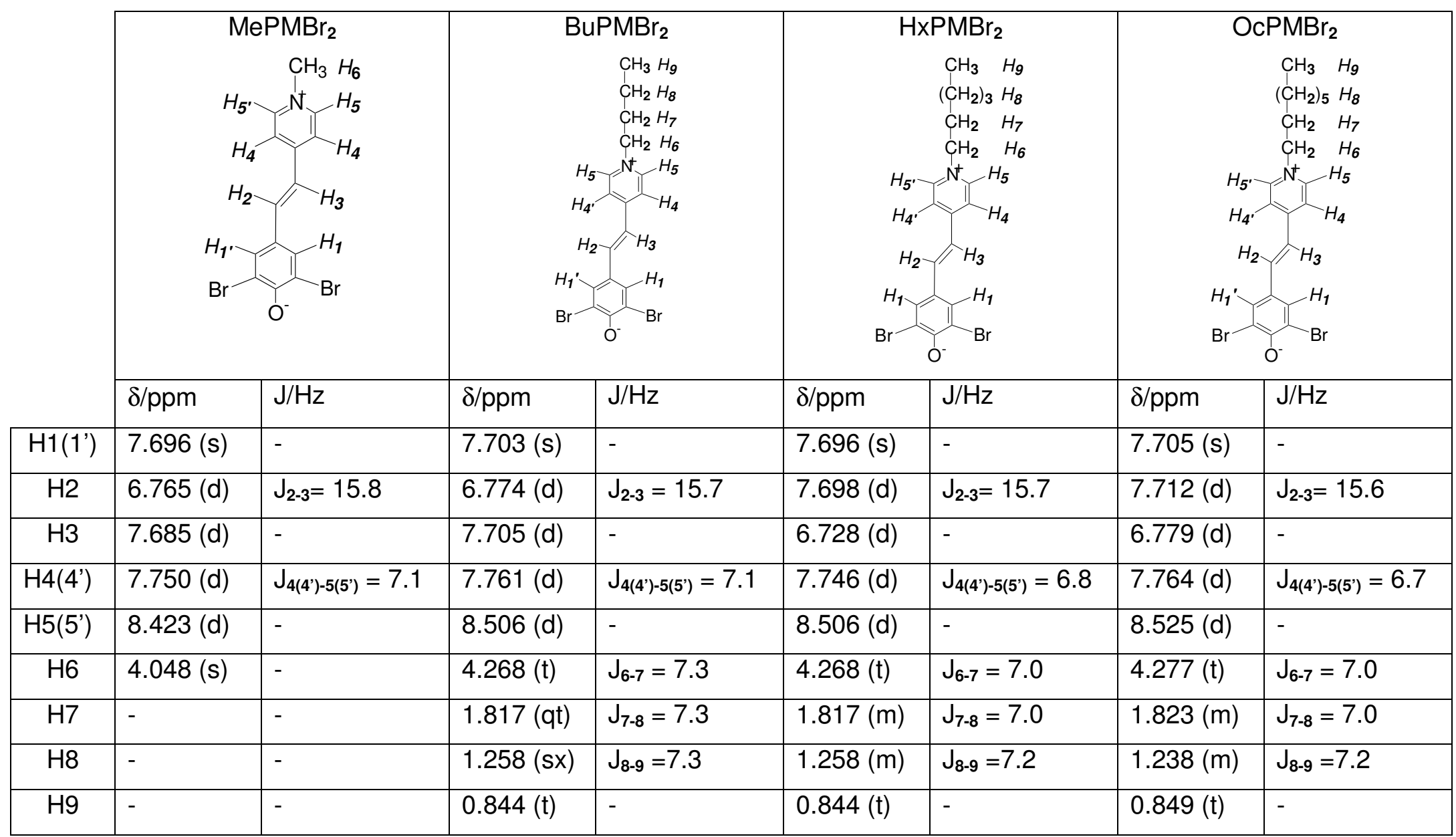

a- At $300 \mathrm{MHz}$ and $25^{\circ} \mathrm{C}$, digital resolution $=0.5 \mathrm{~Hz} /$ data point, solvent DMSO-d6, reference TMS.

b- The following abbreviations were employed for reporting peak multiplicity: $d$, doublet; m, multiplet; qt, quintet; s, singlet; sx, sextet and t, triplet, respectively. 
Table SI-6. Structures, numbering of carbons and ${ }^{13} \mathrm{C}$ NMR data for the probes synthesized. ${ }^{\mathrm{c}}$

\begin{tabular}{|c|c|c|c|c|}
\hline & $\mathrm{MePMBr}_{2}$ & 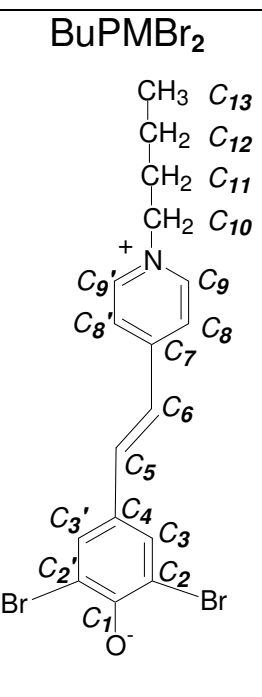 & 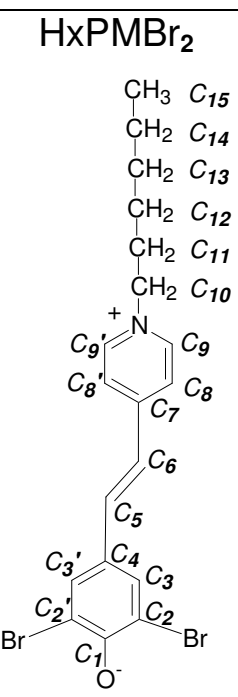 & 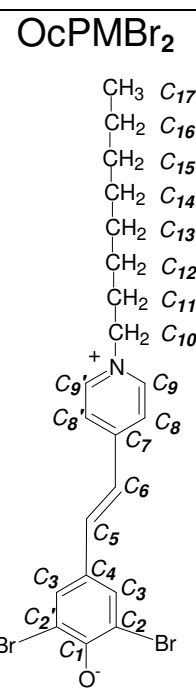 \\
\hline & $\delta / p p m$ & d/ppm & $\delta / p p m$ & $\delta / p p m$ \\
\hline C1 & 163.16 & 165.15 & 165.49 & 165.44 \\
\hline C2(2') & 115.94 & 115.87 & 115.97 & 115.98 \\
\hline C3(3') & 141.50 & 141.73 & 141.83 & 141.83 \\
\hline C4 & 116.75 & 116.47 & 116.26 & 116.23 \\
\hline C5 & 111.80 & 111.97 & 111.83 & 111.83 \\
\hline C6 & 132.72 & 132.70 & 132.79 & 132.73 \\
\hline C7 & 153.53 & 153.80 & 153.86 & 153.82 \\
\hline C8(8') & 120.28 & 120.47 & 120.47 & 120.45 \\
\hline C9(9') & 143.23 & 142.34 & 142.40 & 142.34 \\
\hline C10 & 45.54 & 58.08 & 58.33 & 58.32 \\
\hline C11 & & 32.24 & 30.50 & 31.05 \\
\hline C12 & & 18.69 & 30.25 & 30.30 \\
\hline C13 & & 13.23 & 25.03 & 28.38 \\
\hline C14 & & & 21.79 & 28.28 \\
\hline C15 & & & 13.73 & 25.37 \\
\hline C16 & & & & 21.95 \\
\hline C17 & & & & 13.85 \\
\hline
\end{tabular}

c- At $300 \mathrm{MHz}$ and $25^{\circ} \mathrm{C}$, solvent DMSO-d6, reference TMS. 


\section{CALCULATIONS}

\section{Calculation SI-1: Dependence of $E_{T}(p r o b e)$ on the properties of the solvent}

The solvatochromic parameters and log $P_{\text {solv }}$ required for solving Eqn. (6) were taken from literature. ${ }^{1,2}$ In order to compare the regression coefficients of Eqn. (6) directly, all variables- response and predictors- were standardized by subtracting the mean and dividing by the standard deviation, this results in $\beta_{\text {statistical. }}{ }^{3}$ Regression analysis was carried out by using the Statistica program, StatSoft Inc.

The parameters required to solve Eqn. (14) are $\pi_{\text {mixt }}^{*}, \mathrm{pKa}_{\text {mixt }}, \beta_{\text {mixt }}$ and $\log P_{\text {mixt. }}$ The dipolarity/polarizability and basicity terms $\left(\pi_{\text {mixt }}^{*}\right.$ and $\beta_{\text {mixt }}$, respectively) were those published elsewhere. ${ }^{4,5}$. Similar to Eqn. (6), $\alpha_{\text {mixt }}$ could have been employed as a measure of the "acidity" of the binary mixtures. The determination of $\alpha_{\text {mixt }}$ is, however, not trivial and may require the use of several structurally different indicators, as discussed in details elsewhere ${ }^{6-8}$ Therefore, it is convenient to employ a more fundamental acidity scale, pKa $_{\text {mixt }}$, whose values were experimentally determined for aqueous methanol. ${ }^{9}$. For aqueous $\mathrm{PrOH}$, values of $\mathrm{pKa}_{\text {mixt }}$ were calculated by assuming that the dependence of $\mathrm{pKa}_{\text {mixt }}$ on $\chi_{\mathrm{w}}$ is similar to that of aqueous methanol; the latter is given by Eqn. SI-1 (Equation 1 of Supporting Information 1):

$$
\mathrm{pKa}_{\text {меон-w }}=15.5-4.6221 \chi_{\mathrm{w}}+4.9119 \chi_{\mathrm{w}}{ }^{2}-1.7858 \chi_{\mathrm{w}}{ }^{3}
$$

The pKa of $\mathrm{PrOH}$ (16.1) was substituted for that of $\mathrm{MeOH}$ (15.5); the regression coefficients of the $\chi_{w}$ terms were multiplied by 1.35 in order to obtain the pKa of water (= 14) at $\chi_{w}=1$, i.e.,

$$
\text { pKa ProH-w }=16.1-6.2398 \chi_{w}+6.6311 \chi_{w}{ }^{2}-2.4108 \chi_{w}{ }^{3} \quad \text { (Eqn. SI-2) }
$$

Experimental determination of $\log \mathrm{P}_{\text {mixt }}$ is not feasible. The reason is that the third component (e.g. $\mathrm{PrOH})$ is miscible in both water and n-octanol; this changes the mutual 
solubilities of both phases and may lead to the separation of a third phase. We assumed that the mixture behaves ideally, i.e.,

$$
\log P_{\text {mixt }}=\log P_{w} \chi_{w}+\log P_{\text {ROH }} \chi_{\text {ROH }} \quad \text { (Eqn. SI-3) }
$$

\section{Calculation SI-2: Dissociation constant of Solv-W, $K_{\text {dissoc }}, x_{\text {Species }}^{\text {Effective }}$, and solvent fractionation factors}

Calculation of these parameters has been discussed in details elsewhere, and will be addressed here only briefly. ${ }^{10-12}$ Knowledge of $\mathrm{K}_{\text {dissoc }}$ allows calculation of the effective mole fractions of all solvent species present; this equilibrium constant is calculated from the dependence of binary mixture density, $\rho_{\text {mixt }}$, on its composition, as given by Eqns. (Eqn. SI-4) and (Eqn. SI-5) for Solv/W mixtures: ${ }^{13,14}$

$$
\begin{gathered}
\mathrm{K}_{\text {dissoc }}=\frac{[\text { Solv }]_{\mathrm{Bk} ; \text { Effective }}[\mathrm{W}]_{\mathrm{Bk} ; \text { Effective }}}{[\text { Solv }-\mathrm{W}]_{\mathrm{Bk} ; \text { Effective }}} \quad(\text { Eqn. SI-4) } \\
\rho_{\text {mixt }}=\frac{[\mathrm{W}]_{\mathrm{Bk} ; \text { Effective }} \mathrm{M}_{\mathrm{W}}+[\text { Solv }]_{\mathrm{Bk} ; \text { Effective }} \mathrm{M}_{\text {Solv }}+[\text { Solv }-\mathrm{W}]_{\mathrm{Bk} ; \text { Effective }} \mathrm{M}_{\text {Solv-W }}}{[\mathrm{W}]_{\mathrm{Bk} ; \text { Effective }} \mathrm{V}_{\mathrm{W}}+[\text { Solv }]_{\mathrm{Bk} ; \text { Effective }} \mathrm{V}_{\text {Solv }}+[\text { Solv }-\mathrm{W}]_{\mathrm{Bk} ; \text { Effective }} \mathrm{V}_{\text {Solv }-\mathrm{W}}}(\text { Eqn. SI-5) }
\end{gathered}
$$

Where $\mathrm{M}$ and $\mathrm{V}$ refer to molar mass and volume of the species, respectively. $\mathrm{V}_{\text {Solv-w }}$ may be directly calculated from Eqn. SI-5, by iteration. Alternatively, it may be obtained from $a b$ initio calculations in the gas phase, corrected for temperature and mixture non-ideality, as recently demonstrated for $\mathrm{ROH}-\mathrm{W}$ mixtures. Values of $\mathrm{K}_{\text {dissoc, }} \mathrm{L}$ mol ${ }^{-1}$, employed were: $0.00138,0.00450,0.00461\left(\mathrm{MeOH}, 10,25,40^{\circ} \mathrm{C}\right) ; 0.07522,0.08399,0.09313,0.10436$ $\left.\left(\mathrm{PrOH}, 10,25,40,60^{\circ} \mathrm{C}\right)\right)^{15}(0.08901,0.09923,0.10952,0.12351$ (MeCN, 10, 25, 40, 60 $\left.{ }^{\circ} \mathrm{C}\right) ; 0.00940,0.00963,0.00992\left(\mathrm{DMSO}, 25,40,60^{\circ} \mathrm{C}\right)$. Details of ab-initio calculations of the $\mathrm{V}_{\text {Solv-w }}$ for complexes of MeCN and DMSO will be reported elsewhere. For DMSO at $25^{\circ} \mathrm{C}$, values of $\mathrm{K}_{\text {dissoc }}$ calculated by iteration of Eqn. SI-5 and that based on theoretically calculated $V_{\text {DMSO-w }}$ are in good agreement, 0.009 and 0.0094 , respectively. 
The probe solvation micro-sphere is composed of W, Solv, and Solv-W. Observed $E_{T}, E_{T}^{\text {Obs }}$, is the sum of the polarity of each component, $E_{T}^{W}, E_{T}^{\text {Solv }}, E_{T}^{\text {Solv }-W}$, respectively, multiplied by the corresponding mole fraction in the probe solvation microsphere, $X_{\mathrm{w}}^{\text {Probe }}, X_{\text {Solv }}^{\text {Probe }}$, and $X_{\text {Solv }-\mathrm{w}}^{\text {Probe }}$, respectively:

$$
\mathrm{E}_{\mathrm{T}}^{\text {obs }}=x_{\mathrm{w}}^{\text {Probe }} \mathrm{E}_{\mathrm{T}}^{\mathrm{W}}+x_{\text {Solv }}^{\text {Probe }} \mathrm{E}_{\mathrm{T}}^{\text {Solv }}+x_{\text {Solv }-\mathrm{W}}^{\text {Probe }} \mathrm{E}_{\mathrm{T}}^{\text {Solv-W }} \quad \text { (Eqn. SI-6) }
$$

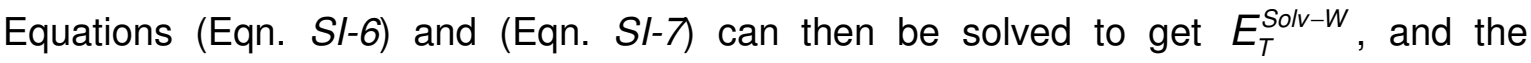
appropriate solvent fractionation factors, respectively.

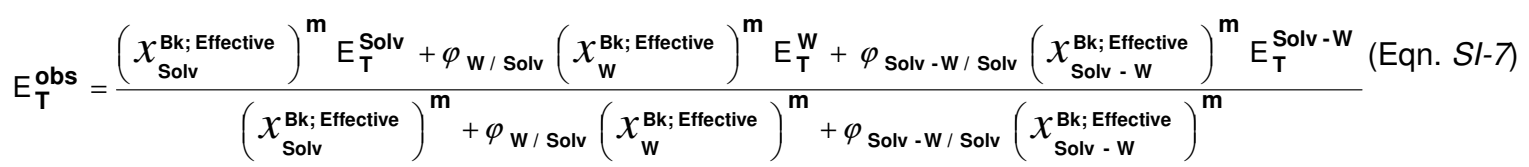

The input data to solve Eqn. SI-7 include $E_{T}^{\text {Obs }}, E_{T}^{W}, E_{T}^{\text {Solv }}$, and $X_{\text {Species }}^{\text {Effective }}$, along with initial estimates of $\mathrm{m}, E_{T}^{\text {Solv }-W}$, and the appropriate $\varphi$. The solvent fractionation factor,

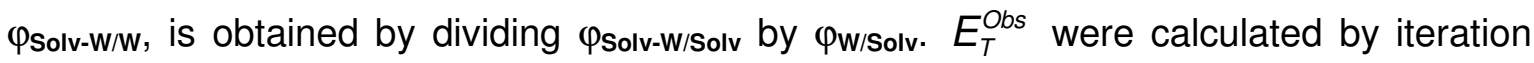
until $\Sigma Q^{2}$ (sum of the squares of the residuals) was not reduced, calculations were carried out by Origin version 6.0, Microcal Co.

\section{References}

(1) Reichardt, C. In Solvents and Solvent Effects in Organic Chemistry; 3rd ed.; VCH: Weinheim, 2003; p 389.

(2) http://www.syrres.com/esc/physdemo.htm

(3) Hill, T.; Lewicki, P. In Statics Methods and Applications, A comprehensive reference for science, industry and data mining; 1st ed.; StatSoft: Tulsa, 2006; pp 555-757.

(4) Wrona, P. K.; Krygowski, T. M.; Zielkowska, U. Z. Naturforsch 1989, 44, 673-678. 
(5) Buhvestov, U.; Rived, F.; Rafols, C.; Bosch, E.; Roses, M. J. Phys. Org. Chem. 1998, $11,185-192$.

(6) Reta, M. R.; Anunziata, J. D.; Cattana, R. I.; Silber, J. J. Anal. Chim. Acta 1995, 306, 81-89.

(7) Cheong, W. J.; Carr, P. W. Anal. Chem. 1988, 60, 820-826.

(8) Park, J. H.; Jang, M. D.; Kim, D. S.; Carr, P. W. J. Chromatogr. 1990, 513, 107-116.

(9) Bosch, E.; Bou, P.; Alleman, H.; Roses, M. Anal. Chem. 199, 68, 3651-3657.

(10) Tada, E. B.; Silva, P. L.; El Seoud, O. A. J. Phys. Org. Chem. 2003, 16, 691-699.

(11) Tada, E. B.; Silva, P. L.; El Seoud, O. A. Phys. Chem. Chem. Phys. 2003, 5, 53785385.

(12) Tada, E. B.; Silva, P. L.; Tavares, C.; El Seoud, O. A. J. Phys. Org. Chem. 2005, 18, 398-407.

(13) Katz, E. D.; Lochmuller, C. H.; Scott, R. P. W. Anal. Chem. 1989, 61, 349-355.

(14) Scott, R. P. W. Analyst 2000, 125, 1543-1547.

(15) Bastos, E. L.; Silva, P. L.; El Seoud, O. A. J. Phys. Chem. A 2006, 110, 1028710295. 